\title{
PROJECTIVE BITOPOLOGICAL SPACES
}

\author{
M. C. DATTA \\ (Received 15 October 1969; revised 4 February 1970) \\ Communicated by B. Mond
}

\section{Introduction}

J. C. Kelly [2] introduced the concept of a bitopological space. Lane [3], Patty [4] and Pervin [5] have continued his work. Our purpose in this paper is to identify the projective objects in a suitable category of bitopological spaces after the manner of Gleason [1] and generalize his theorem that in the category of compact Hausdorff topological spaces, the projective spaces are precisely the extremally disconnected ones.

We give new definitions of continuity and of separation in bitopological spaces. These definitions are strictly weaker than the corresponding ones (for bitopological spaces) in the literature. Precisely, our 'quasi-continuity' is weaker than Pervin's continuity [5] and our 'quasi-Hausdorff' bitopological space is more general than a pairwise Hausdorff one [2]. Here we give a definition of 'semicompactness' for bitopological spaces. (The reason for the nomenclature will be clear from the definition.) All these three concepts reduce to the corresponding conventional concepts, with the prefix 'quasi' or 'semi' removed, when the two topologies of the bitopological structure coincide and we have just a space with a single topology. We also generalize the definition of an extremally disconnected space to bitopological space. Under this setting, we prove the following

MAIN THEOREM. In the category of semi-compact, quasi-Hausdorff bitopological spaces and quasi-continuous maps, the projective spaces are precisely the extremally disconnected ones.

Throughout the following, $(X, P, Q)$ stands for a bitopological space with topologies $P$ and $Q$. If we need two bitopological spaces simultaneously we shall take them as $\left(X_{1}, P_{1}, Q_{1}\right)$ and $\left(X_{2}, P_{2}, Q_{2}\right)$.

\section{Preliminaries on continuity}

2.1. Definition. A subset $A$ of $(X, P, Q)$ is said to be quasi-open if for every $x \in A$ there exists a $P$-open neighborhood $U_{x} \subset A$ or a $Q$-open neighbourhood $V_{x} \subset A$. 
2.2. Proposition. Quasi-open sets are precisely the unions of $P$-open and $Q$ open sets.

Proof follows directly from definitions.

REMARK. Every $P$-open ( $Q$-open) set is quasi-open. That arbitrary union of quasi-open sets is quasi-open follows by 2.2. Finite intersection of quasi-open sets need not be quasi-open. For example, let $X$ be the real line $R$. Let $P$ be the topology with base $[a, b)$ and $Q$ be the topology with base $(c, d]$. Let $a<b<c$. Then $(a, b]$ and $[b, c)$ are quasi-open sets, but $(a, b] \cap[b, c)=\{b\}$ is not a quasiopen set.

2.3. Definition. A quasi-closed set is the complement of a quasi-open set.

Remark. Every $P$-closed ( $Q$-closed) set is quasi-closed. Arbitrary intersection of quasi-closed sets is quasi-closed. Finite union of quasi-closed sets need not be quasi-closed. Every quasi-closed set is the intersection of a $P$-closed set and a $Q$ closed set.

2.4. Definttion. The quasi-closure of $A \subset(X, P, Q)$ is $(P-\operatorname{cl}(A)) \cap(Q-\operatorname{cl}(A))$, where $P$-cl denotes $P$-closure and $Q$-cl denotes $Q$-closure. The quasi-closure of $A$ is denoted by $\bar{A}$.

2.5. Proposition. If $A \subset(X, P, Q)$, then $\bar{A}$ is the smallest quasi-closed set containing $A$.

Proof follows from definitions 2.1, 2.3 and 2.4.

2.6. Definition. Let $f:\left(X_{1}, P_{1}, Q_{1}\right) \rightarrow\left(X_{2}, P_{2}, Q_{2}\right) . f$ is said to be quasicontinuous if the inverse image of every quasi-open set is quasi-open.

2.7. Proposition. Let $f:\left(X_{1}, P_{1}, Q_{1}\right) \rightarrow\left(X_{2}, P_{2}, Q_{2}\right)$ be quasi-continuous. Let $G \subset X_{1}$. Then $f(\bar{G}) \subset \overline{f(G)}$.

ProOF. Let $x \in\left(P_{1}-\operatorname{cl}(G) \cap Q_{1}-\operatorname{cl}(G)\right)$. Let $U$ be any $P_{2}$-neighborhood of $f(x)$ in $X_{2}$. Since $f$ is quasi-continuous, $f^{-1}(U)$ is quasi-open in $X_{1}$ and $x \in f^{-1}(U)$. Therefore there exists either a $P_{1}$-neighborhood or a $Q_{1}$-neighborhood $W$ of $x$ such that $W \subset f^{-1}(U)$. $W$ meets $G$ because $x \in\left(P_{1}-\operatorname{cl}(G) \cap Q_{1}-\operatorname{cl}(G)\right)$. Hence $f^{-1}(U)$ meets $G$ and so $U$ meets $f(G)$. Hence $f(x) \in P_{2}-\operatorname{cl}(f(G))$. Similarly $f(x) \in Q_{2}-\operatorname{cl}(f(G))$. Thus $f(\bar{G}) \subset \overline{f(G)}$.

REMARK. Every quasi-continuous map is continuous for the upper bound topology. The converse need not be true as is shown by the following example.

Let $X_{1}$ be the real line $R$ and $d$ be a metric defined by $d(x, y)=\min$. $\{1,|x-y|\}$. Define conjugate quasi-pseudo-metrics $p$ and $q$ on $X_{1}$ by putting, for $(x, y) \in R$,

$$
p(x, y)= \begin{cases}d(x, y) & \text { if } x \leqq y \\ 1 & \text { if } x>y\end{cases}
$$

and $q(x, y)=p(y, x)$. 
Let $P_{1}$ and $Q_{1}$ be the topologies on $X_{1}$ determined by $p$ and $q$ respectively. Let $X_{2}$ be the real line $R$. Define $u(x, x)=0$ for each $x \in R$. If $x \neq y$, define $u(x, y)=1$ if $x$ is rational and $u(x, y)=|x-y| /(1+|x-y|)$ if $x$ is irrational. Define $v$ by $v(x, y)=u(y, x)$. Let $P_{2}$ and $Q_{2}$ be the topologies on $X_{2}$ determined by $u$ and $v$ respectively.

Consider the identity map $I:\left(X_{1}, P_{1}, Q_{1}\right) \rightarrow\left(X_{2}, P_{2}, Q_{2}\right) . I$ is continuous in the upper bound topology. $I$ is not quasi-continuous. For, consider the open interval $(a, b)$ without rational points. It is quasi-open in $X_{2}$ but is not quasiopen in $X_{1}$.

\section{Semi-compact bitopological spaces}

3.1. Definition. $A \subset(X, P, Q)$ is said to be semi-open if it is open in the upper bound topology of $P$ and $Q$.

Remark. Consequently we have the natural notion of a semi-closed set and of semi-closure.

3.2 Definition. A set $A \subset(X, P, Q)$ is said to be semi-compact if it is compact in the upper bound topology of $P$ and $Q$; in other words $A$ is semi-compact if and only if, given any covering of $A$ by semi-open subsets of $X$ there exists a finite subcovering.

Remark. Every semi-closed (and therefore every $P$-closed, $Q$-closed and quasi-closed) subset of a semi-compact bitopological space is semi-compact.

3.3. Proposition. The quasi-continuous image of a semi-compact bitopological space is semi-compact.

Proof. Let $f$ be a quasi-continuous mapping of a semi-compact space $\left(X_{1}, P_{1}, Q_{1}\right)$ onto an arbitrary space $\left(X_{2}, P_{2}, Q_{2}\right)$. We shall show that $\left(X_{2}, P_{2}, Q_{2}\right)$ is semi-compact.

Let $v=\left(U_{\alpha}\right)_{\alpha \in A}$ be a covering of $X_{2}$, where each $U_{\alpha}$ is semi-open and so of the form $\bigcup_{i, j}\left(V_{\alpha_{i}} \cap W_{\alpha_{j}}\right)$ where $V_{\alpha_{i}}$ is $P$-open and $W_{\alpha_{j}}$ is $Q$-open. Then $f^{-1}\left(U_{\alpha}\right)=$ $f^{-1}\left(\bigcup_{i, j}\left(V_{\alpha_{i}} \cap W_{\alpha_{j}}\right)\right)=\bigcup_{i, j}\left(f^{-1}\left(V_{\alpha_{i}}\right) \cap f^{-1}\left(W_{\alpha_{j}}\right)\right) . \quad V_{\alpha_{i}}$ is quasi-open, so $f^{-1}\left(V_{\alpha_{i}}\right)$ is quasi-open and hence semi-open. Similarly, $f^{-1}\left(W_{\alpha_{j}}\right)$ is semi-open. Hence $f^{-1}\left(U_{\alpha}\right)$ is semi-open. Therefore $\left(f^{-1}\left(U_{\alpha}\right)\right)_{\alpha \in A}$ is a semi-open covering of $X_{1}$. Since $X_{1}$ is semi-compact, there exists a finite subset $A_{1} \subset \Lambda$ such that $\bigcup_{\alpha \in A_{1}} f^{-1}\left(U_{\alpha}\right)$ covers $X_{1}$. Therefore $\bigcup_{\alpha \in A_{1}} U_{\alpha}$ is a finite subcovering of $v$ which covers $X_{2}$. Therefore $X_{2}$ is semi-compact.

3.4. Definition. $(X, P, Q)$ is said to be quasi-Hausdorff if given $x_{1} \neq x_{2}$ there exist quasi-open sets $U_{1}, U_{2}$ such that $x_{1} \in U_{1}, x_{2} \in U_{2}$ and $U_{1} \cap U_{2}=\emptyset$.

Remark. Kelly [2] calls $(X, P, Q)$ pairwise Hausdorff if given $x_{1} \neq x_{2}$ there exists a $P$-open set $U_{1}$ and a $Q$-open set $U_{2}$ containing $x_{1}$ and $x_{2}$ respectively such 
that $U_{1} \cap U_{2}=\emptyset$. So every pairwise Hausdorff space is also quasi-Hausdorff. But the converse need not be true. For example, let $X$ be the real line $R$ and $d$ be a metric defined by $d(x, y)=\min$. $\{1,|x-y|\}$. Define, for $(x, y) \in R, u(x, y)=0$ if $x \leqq y,=d(x, y)$ if $x>y$ and $v(x, y)=u(y, x)$.

Let $P$ and $Q$ be the topologies on $X$ determined by $u$ and $v$ respectively. $(X, P, Q)$ is quasi-Hausdorff but not pairwise Hausdorff.

3.5. Proposition. Let $(X, P, Q)$ be a quasi-Hausdorff space. Let $U_{1}$ and $U_{2}$ be quasi-open sets such that $U_{1} \cap U_{2}=\emptyset$. Then $\bar{U}_{1} \cap U_{2}=\emptyset$.

Proof. Let $U_{1}$ and $U_{2}$ be quasi-open sets such that $U_{1} \cap U_{2}=\emptyset$. We shall show that $\bar{U}_{1} \cap U_{2}=\emptyset$. Suppose $\bar{U}_{1} \cap U_{2} \neq \emptyset$. Let $y \in \bar{U}_{1} \cap U_{2} . y \in \bar{U}_{1}$ implies every $P$-neighborhood and $Q$-neighborhood of $y$ meets $U_{1}$. But $U_{2}$ is a quasiopen set containing $y$ and so there exists either a $P$-neighborhood or a $Q$-neighborhood $W$ of $y$ such that $W \subset U_{2}$. But by hypothesis $U_{2}$ does not meet $U_{1}$, so $W \cap U_{1}=\emptyset$. This contradiction establishes that $\bar{U}_{1} \cap U_{2}=\emptyset$.

3.6. Definition. Let $\left(X_{i}, P_{i}, Q_{i}\right)_{i \in I}$ be a family of bitopological spaces. On the product set $X=\prod_{i \in I} X_{i}$, we define a bitopological structure $(P, Q)$ by taking $P$ as the product topology generated by the $P_{i} s$ and $Q$ as the product topology generated by the $Q_{i} s$.

3.7. Proposition. The natural projection from a product bitopological space $(X, P, Q)$ on the component spaces $\left(X_{i}, P_{i}, Q_{i}\right)_{i \in I}$ is quasi-continuous.

Proof. Let $\prod_{i}$ denote the projection from $X$ onto $X_{i}$. Let $U$ be any quasiopen set of $X_{i}$. Then $U=V \cup W$ where $V$ is $P_{i}$-open and $W$ is $Q_{i}$-open. $\prod_{i}^{-1}(U)=$ $\prod_{i}^{-1}(V) \cup \prod_{i}^{-1}(W) . \prod_{i}^{-1}(V)$ is $P$-open and $\prod_{i}^{-1}(W)$ is $Q$-open. Therefore $\prod_{i}^{-1}(U)$ is quasi-open in $X$. Hence $\prod_{i}$ is quasi-continuous.

3.8. Proposition. The product of any arbitrary number of semi-compact bitopological spaces is semi-compact.

Proof. Let $\left(X_{i}, P_{i}, Q_{i}\right)_{i \in I}$ be a family of semi-compact spaces. Let $\tau_{i}$ be the upper bound topology of $P_{i}$ and $Q_{i}$. Let $(X, \tau)=\prod_{i \in I}\left(X_{i}, \tau_{i}\right)$ be the product space, and let $(X, P, Q)=\prod_{i \in I}\left(X_{i}, P_{i}, Q_{i}\right)$ be the product bitopological space. Then $(X, \tau)$ is compact since by definition each $\left(X_{i}, \tau_{i}\right)$ is compact. Since $\tau$ is finer than the upper bound topology of $P$ and $Q,(X, P, Q)$ is semi-compact.

3.9. Proposition. Let $\left(A, P_{1}, Q_{1}\right),\left(B, P_{2}, Q_{2}\right)$ and $\left(C, P_{3}, Q_{3}\right)$ be quasiHausdorff spaces. Let $f: B \rightarrow C$ and $g: A \rightarrow C$ be quasi-continuous maps. Then $D=\{(a, b): g(a)=f(b)\}$ is a semi-closed subset of $A \times B$.

Proof. Let $(A \times B, P, Q)$ be the product space of $\left(A, P_{1}, Q_{1}\right)$ and $\left(B, P_{2}, Q_{2}\right)$. Let $\left(a_{1}, b_{1}\right) \in(A \times B)-D$. Therefore $g\left(a_{1}\right) \neq f\left(b_{1}\right)$ in $C$. Because $C$ is quasi-Hausdorff, there exists disjoint quasi-open sets $U_{1}$ and $U_{2}$ containing 
$g\left(a_{1}\right)$ and $f\left(b_{1}\right)$. So, $a_{1} \in g^{-1}\left(U_{1}\right)$ and $b_{1} \in f^{-1}\left(U_{2}\right)$. Since $g$ and $f$ are quasicontinuous, $g^{-1}\left(U_{1}\right)$ and $f^{-1}\left(U_{2}\right)$ are quasi-open sets in $A$ and $B$ respectively. Since a quasi-open set is also semi-open, $g^{-1}\left(U_{1}\right) \times f^{-1}\left(U_{2}\right)$ is a semi-open subset of $A \times B$.

We shall now show that $D \cap\left\{g^{-1}\left(U_{1}\right) \times f^{-1}\left(U_{2}\right)\right\}=\emptyset$. Suppose these two sets are not disjoint, then let $\left(a_{2}, b_{2}\right) \in D \cap\left\{g^{-1}\left(U_{1}\right) \times f^{-1}\left(U_{2}\right)\right\} .\left(a_{2}, b_{2}\right) \in D$ implies $g\left(a_{2}\right)=f\left(b_{2}\right) .\left(a_{2}, b_{2}\right) \in\left\{g^{-1}\left(U_{1}\right) \times f^{-1}\left(U_{2}\right)\right\}$ implies $g\left(a_{2}\right) \in U_{1}$ and $f\left(b_{2}\right) \in U_{2}$. So, $g\left(a_{2}\right)=f\left(b_{2}\right) \in U_{1} \cap U_{2}$ contradicting the fact that $U_{1}$ and $U_{2}$ are disjoint. Therefore $\left\{g^{-1}\left(U_{1}\right) \times f^{-1}\left(U_{2}\right)\right\}$ is disjoint from $D$.

Thus for every point $\left(a_{1}, b_{1}\right) \in(A \times B)-D$ there exists a semi-open set disjoint from $D$. Therefore $(A \times B)-D$ is semi-open and hence $D$ is semi-closed.

\section{Extremally disconnected bitopological spaces}

4.1. Definition. $(X, P, Q)$ is said to be extremally disconnected if for every semi-open set its quasi-closure is quasi-open.

4.2. Proposmion. A semi-compact subset of a quasi-Hausdorff bitopological space is semi-closed.

This is the analogue for bitopological spaces of the classical result that a compact subset of a Hausdorff space is closed. The proof runs on the same pattern. We have only to recall that every quasi-open set is semi-open.

However this proposition is not enough for the proof of our main theorem. So we prove the following

4.3. Proposition. A semi-compact subset of a quasi-Hausdorff bitopological space which is also extremally disconnected is quasi-closed.

REMARK. The hypothesis of extremally disconnectedness is not necessary for the conclusion as is shown by the space $(X, P, Q)$ of 3.3 (Remark). A semi-compact subset here is a closed bounded interval $[a, b]$ which is quasi-closed in $X$. But we do not know how to prove the proposition without this extra hypothesis.

Proof of the Proposition. Let $A$ be a semi-compact subset of a quasi-Hausdorff space $(X, P, Q)$ which is also extremally disconnected. We shall show that $X-A$ is quasi-open.

Let $s \in X-A$. By proposition 3.5, there exist for each $a \in A$ disjoint quasiopen sets $U_{a}$ and $V_{a}$ containing $a$ and $s$ respectively such that $U_{a} \cap \bar{V}_{a}=\emptyset$. The collection $\left\{U_{a}\right\}_{a \in A}$ is a semi-open covering of $A$. By compactness of $A$, there exists a finite subset $A_{1} \subset A$ such that $\bigcup_{a \in A_{1}} U_{a}$ covers $A$. Thus $A \subset \bigcup_{a \in A_{1}} U_{a}$. Let $W_{s}=\bigcap_{a \in A_{1}} \bar{V}_{a}$. Then $\overline{\bigcap_{a \in A_{1}} V_{a}} \subset \bigcap_{a \in A_{1}} \bar{V}_{a}=W_{s}$. But $\overline{\bigcap_{a \in A_{1}} V_{a}}$ is quasi-open (because $(X, P, Q)$ is extremally disconnected). So $W_{s}$ contains a quasi-open set containing $s$. Also $A \cap W_{s} \subset\left(\bigcup_{a \in A_{1}} U_{a}\right) \cap W_{s}=\emptyset$. Thus for each $s \in X-A$, there exists a quasi-open set $\subset W_{s}$ such that $W_{s} \cap A=\emptyset$. This proves $X-A$ is quasi-open. Hence $A$ is quasi-closed. 


\section{Generalization of Gleason's lemmas}

5.1. LEMMA. Let $\rho:(E, P, Q) \rightarrow\left(A_{1}, P_{1}, Q_{1}\right)$ be quasi-continuous and onto such that $\rho\left(E_{0}\right) \neq A$ for any proper semi-closed subset $E_{0} \subset E$. Then for any quasi-open set $G \subset E, \rho(G) \subset \overline{(A-\rho(E-G))}$.

The proof runs on the same lines as that of lemma 2.1 of [1].

5.2. LEMMA. In an extremally disconnected quasi-Hausdorff bitopological space, if $U_{1}$ and $U_{2}$ are two disjoint quasi-open sets then $\bar{U}_{1} \cap \bar{U}_{2}=\emptyset$.

The proof follows from proposition 3.5 and definition 4.1.

5.3. LeMMA. Let $(E, P, Q)$ be a semi-compact space and $\left(A, P_{1}, Q_{1}\right)$ be a quasi-Hausdorff and extremally disconnected space. Let $\rho: E \rightarrow A$ be quasi-continuous, one-one and onto. Then $\rho^{-1}$ is quasi-continuous.

Proof. Let $F$ be a quasi-closed subset of $E$. Then $F$ is semi-compact. Since $\rho$ is quasi continuous, $\rho(F)$ is semi-compact. So, by proposition $4.3, \rho(F)$ is quasiclosed in $A$. This proves $\rho^{-1}$ is quasi-continuous.

5.4 LemMA. Let $(E, P, Q)$ be semi-compact and quasi-Hausdorff, and $\left(A, P_{1}, Q_{1}\right)$ be semi-compact, quasi-Hausdorff and extremally disconnected spaces. Let $\rho: E \rightarrow A$ be quasi-continuous and onto. Let $\rho\left(E_{0}\right) \neq A$ for any proper semi-closed subset $E_{0} \subset E$. Then $\rho$ is $1-1$ and $\rho^{-1}$ is quasi-continuous.

PRoOF. In view of Lemma 5.3 we have only to prove that $\rho$ is one-one. The proof of this follows on the same lines as that of Lemma 2.3 of [1].

5.5. Lemma. Let $(A, P, Q)$ and $\left(D, P_{1}, Q_{1}\right)$ be semi-compact, quasi-Hausdorff spaces. Let $\prod: D \rightarrow A$ be quasi-continuous and onto. Then $D$ contains a semicompact subset $E$ such that $\Pi(E)=A$ but $\Pi\left(E_{0}\right) \neq A$ for any proper semi-closed subset $E_{0} \subset E$.

The proof runs along the same lines as that of lemma 2.4 of [1].

\section{Proof of the main theorem}

Let us first prove that every extremally disconnected semi-compact quasiHausdorff space $(A, P, Q)$ is projective. Let $\left(B, P_{1}, Q_{1}\right)$ and $\left(C, P_{2}, Q_{2}\right)$ be semicompact quasi-Hausdorff spaces and $f: B \rightarrow C$ and $g: A \rightarrow C$ be quasi-continuous maps and $f$ is also onto.

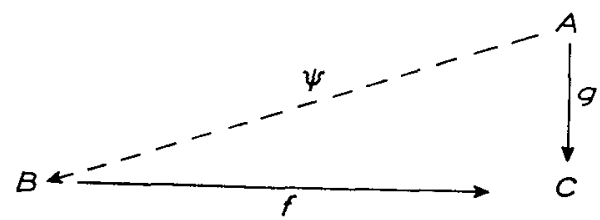

We have to produce a quasi-continuous map $\psi: A \rightarrow B$ such that the above diagram commutes. Consider $A \times B$ and its subset $D=\{(a, b): g(a)=f(b)\}$. 
By proposition 3.8, $D$ is semi-closed subset of $A \times B$. By proposition 3.7, $A \times B$ is semi-compact and hence $D$ is semi-compact. Consider the projection $\prod_{1}: A \times B \rightarrow A$. Since $f$ is onto, $\{f(b): b \in B\}$ exhausts $C$ and therefore $\{g(a)$ : $(a, b) \in D\}$ exhausts $C$ which means $\prod_{1}$ maps $D$ onto $A$. By Lemma 5.5 , there exists a semi-compact subset $E \subset D$ such that $\prod_{1}(E)=A$ but $\prod_{1}\left(E_{0}\right) \neq A$ for any proper semi-closed subset $E_{0} \subset E$. Let $\rho=\prod_{1} E$. $\rho$ satisfies all the conditions of Lemma 5.4 and so $\rho^{-1}$ exists and is quasi-continuous. Let

$$
\psi=\prod_{2} \circ \rho^{-1}: A \stackrel{\rho^{-1}}{\longrightarrow} E \subset A \times B \stackrel{\Pi_{2}}{\longrightarrow} B .
$$

$\psi$ is quasi-continuous. We shall show that $\psi$ is the required map. Let $a \in A$. Since $\rho^{-1}(a) \in D$,

$$
f\left(\prod_{2}\left(\rho^{-1}(a)\right)\right)=g\left(\prod_{1}\left(\rho^{-1}(a)\right)\right)=g(a) .
$$

Therefore $g=f \circ \prod_{2} \circ \rho^{-1}=f \circ \psi$. This proves one part of the theorem.

Now let us prove that every projective space is extremally disconnected.

Let $(X, P, Q)$ be a projective space. Let $G$ be semi-open subset of $X$. We shall show that $\bar{G}$ is quasi-open. Consider

$$
Y=((X-G) \times\{p\}) \cup(\bar{G} \times\{q\}) \subset X \times\{p, q\},
$$

where $\{p, q\}$ has the bitopological structure $(\emptyset,\{p\},\{p, q\})$ and $(\emptyset,\{q\},\{p, q\})$.

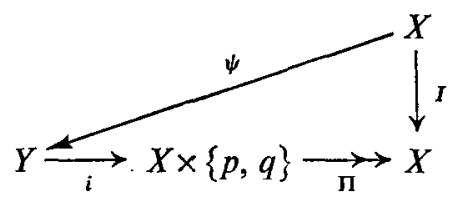

Since $X$ is projective there exists a quasi-continuous map $\psi: X \rightarrow Y$ such that the above diagram commutes, where $I$ is the identity map from $X$ into $X$. $\prod \circ i$ is oneone on $(G \times\{q\})$. Therefore $\psi(x)=(x, q)$ for all $x \in G$. Since $\psi$ is quasi-continuous $\psi(x) \in \overline{(G \times\{q\})}$ for all $x \in \bar{G}$. But $\overline{(G \times\{q\})} \subset \bar{G} \times\{q\}$. Therefore $\psi^{-1}(\bar{G} \times\{q\} \supset \bar{G}$. Also if $x \notin \bar{G}, \psi(x)=(x, p)$. So $\psi^{-1}(\bar{G} \times\{q\})=\bar{G}$. But $\psi$ is quasi-continuous and $(X-G \times\{p\})$ is quasi-closed in $Y$ and so $\bar{G} \times\{q\}$ is quasi-open in $Y$. Therefore $\bar{G}$ is quasi-open in $X$.

REMARK. In the second part of the proof of the theorem the quasi-Hausdorff and semi-compact property of the spaces was not used and so in the category of all bitopological spaces and quasi-continuous maps every projective space is extremally disconnected.

I express my deep sense of gratitude to Prof. V. Krishnamurthy for his encouragement and help in the preparation of this paper. I also thank the referee for his suggestions. 


\section{References}

[1] A. M. Gleason, 'Projective topological spaces', Illi. J. Math. 2 (1958), 482-489.

[2] J. C. Kelly, 'Bitopological spaces', Proc. Lond. Math. Soc. (3) 13 (1963), 71-89.

[3] E. P. Lane, 'Bitopological spaces and quasi-uniform spaces', Proc. Lond. Math. Soc. (3) 17 (1967), 241-256.

[4] C. W. Patty, 'Bitopological spaces', Duke Math. Journ. 34 (1967), 387-391.

[5] W. J. Pervin, 'Connectedness in bitopological spaces', Ind. Math. 29 (1967), 369-372.

\section{Department of Mathematics}

Birla Institute of Technology and Science

Pilani (Rajasthan), India 